\title{
Fluid (in)form and the encoding of space
}

\author{
J. Maze, M. McGlothlin \& K. Tanzer \\ School of Architecture, University of Florida, USA
}

\begin{abstract}
The architect is faced with a multifaceted design problem when designing the roof, particularly where rain is concerned. The roof must be load bearing, supporting not only its own weight, but also that of any collecting water or snow. In an area with a severe rainy season such as Florida, this problem is magnified exponentially. This project addresses the nature of water as it interacts with a roof form by testing various roof designs and pitches, and helps educate the design students to the essential needs in the design of a roof system. Through the benefit of experience, an architect or educator can use intuition and experience to be able to point to a roof portion and say that water will collect here inappropriately. However, it is something completely different for the architect or student to be able to test their theory and see water actually collect on their architectural model and infiltrate their design through the use of computer animation. This paper outlines the experiment with rain simulation, presents the outcomes, and critiques the interdisciplinary relationship between disparate bodies of researchers and students involved.

Keywords: digital modelling, computer simulation, design education, particle systems.
\end{abstract}

\section{Premise}

"My earliest childhood memories are related to a ranch my family owned near the village of Mazamitla. It was a pueblo with hills, formed by houses with tile roofs and immense eaves to shield passers-by from the heavy rains which fall in that area. Even the earth's colour was interesting because it was red earth. In this village, the water distribution system consisted of great gutted logs, in the form of troughs, which ran on a support structure of tree forks, five meters high, above the roofs. The aqueduct crossed over the town, reaching the patios, where there were great stone fountains to receive the water. The patios outside the 
stables, with cows and chickens, all together. Outside, in the street, there were iron rings to tie the horses. The channeled logs, covered with moss, dripped water all over town, of course. It gave this village the ambience of a fairy tale" [1].

The early work of Christopher Alexander and Nicholas Negroponte marked the beginning of architects' selective flirtation with computational technologies. In recent years work developed in the context of Columbia's Paperless Studio and its spin-offs has embraced the fluid form-making potential of programs such as Maya in an effort to subvert the strictures of Cartesian space. This group has also challenged the authorial will of the individual architect by assigning spatial and temporal properties to abstract concepts and setting these now-spatialized concepts in motion. The resulting interaction produces a collision of amorphous forms, currently described colloquially as "blobs."

Our project takes the same issues - the quest for an appropriate fit between computational promise and form-making and a fascination with shaping complex flows - in a different direction. We pose this architectural question: How does architecture shape and redirect one of the earth's physical "flows" - water - as it falls in the form of rain? Can we model rainfall digitally, and then shape surfaces such as roofs, terraces or landscapes to design the flow of water? Can this flow be conceived as a design opportunity, contributing to the phenomenal experience of a project as it is simultaneously shed more responsibly to the larger landscape? Can the poetic exploration of fluids prevalent within the academy be transformed into equally poetic tangible applications?

These are some of the questions we set out to explore, working in collaboration with colleagues in the University of Florida Global Information Systems Center and Digital Worlds Institute (DW). Fifteen students and three faculty members culled from our senior architecture studio met over a period of several weeks with faculty and graduate students with expertise in urban planning, engineering and fine arts. First our architecture students were required to develop a rudimentary understand of GIS regional mapping capacity, with a particular emphasis on the study of hydrology and watersheds across our watery state of Florida. Following this regional analysis we developed a method to demonstrate the water shed by individual student's projects set within this larger landscape. Students first translated their physical models of modest (1000 sf) projects into three-dimensional digital models, then into animations to which rain could be "applied." Students' representations moved from chalk pastel to GIS to basswood to AutoCAD to 3D Studio Max and finally to Maya. Difficulties in translation and changes in material behaviors occurred at each state, and form the primary focus of this paper.

\subsection{Precedents}

Since Renaissance theorists Alberti and Brunelleschi developed a systematic process to construct three-dimensional perspectives on a two-dimensional plane, architects and theorists have forayed into various emergent technologies of spatial representation. Rene Descartes used algebra to develop a coordinate system that removed the necessity of tools or even reference to the real world. 
Nearly four hundred years later, during the 1980s, a new type of spatial research and system emerged in the guise of virtual reality. Early attempts to create a multi-sensual virtual world required a pair of goggles, headphones, and a glove so that one could see, hear, and feel objects modelled in a computer environment. These interfaces were quite cumbersome, and of course inconvenient if one were to imagine working with such a situation on a daily basis. However, they did set the foundation for countless later attempts at designing an artificial world, a cyberspace as described by William Gibson in Neuromancer:

"...cyberspace has a spatial and architectural form that is dematerialized, dynamic and devoid of the laws of physics; spaces in which the mind can explore free of the body; spaces that are in every way socially constructed, produced and abstract. As such, the architecture of cyberspace only mirrors that of Cartesian logic if that is how we straitjacket it" [2].

Arguably, a digital aesthetic has emerged in the architectural world. Those who have begun to explore the notion of an indigenous digital architectural language have come to utilize similar design elements, forms, and spaces in their work. Offices such as the UNstudio, Asymptote, Greg Lynn Form, Architectonics, and Diller-Scofidio who strive to find the architecture of cyberspace instead of simulating the architecture of the physical world have published widely their flowing, transparent, layered projects that seem quite as home in a molecular biology experiment as in a design studio context. Entranced by the idea of interconnectivity, speed, efficiency, and spatial dynamics, these designers began to explore forms only possible through the use of computer modelling technology. New formal typologies continue to emerge that challenges our understanding of the built and designed environment. As these projects begin to be built, often requiring new technologies of construction and fabrication, we start to understand the physical inhabitation of such dynamic environments as being quite different than those of the parallel and angular.

Since the early 1990s, dynamic digital forms have proliferated in graduate schools across the world, thanks largely to the efforts of young faculty members at schools such as Columbia University, Princeton, Cooper Union, UCLA, and Sci-Arc. What is important to note is that by the early nineties, graduates and recent graduates architects were the first generation to not have been educated by the giants of modernism, such as Le Corbusier, Kahn, Aalto, and Mies. The tenets of modernism were distilled through an older generation of faculty, who themselves were exploring ways to expand upon the dogma of their teachers. Hungry for a new language of design, a new way to understand urban form and space, this new generation looked to the digital revolution for inspiration.

"Liquid architecture is an architecture that breathes, pulses, leaps as one form and lands as another. Liquid architecture is an architecture whose form is contingent on the interests of the beholder; it is an architecture that opens to welcome me and closes to defend me; it is an architecture without doors and hallways, where the next room is always where I need it to be and what I need it to be" [3]. 
Perhaps the most noted innovator of digital architecture is Greg Lynn. Lynn began adapting software to the design of complex conceptual models in the late 1980s while at Eisenman Architects, and has continued to evolve his practice and research along with the digital explosion of the 1990s. What Lynn professes to do is typical of his generation - mapping contextual and programmatic forces of a project then modelling them in a way that allows the architecture to grow directly from the data. In this sense, the will of the architect is removed from the equation, and the design is autogenerative. For instance, he uses software such as Maya to create particle flows that simulate pedestrian and vehicular traffic in his entry for the Port Authority Gateway Competition. Particles are dispersed along routes of traffic responding to gravity and one another to create a "phase portrait" [4]. Architectural form is then essentially derived directly from the vectors describing the motion of the particles. Many of the current generation of architects using this technology to create their formal language operate in this manner, allowing the software to create the architecture rather than simply to inform it.

One of the biggest problems with this type of design pedagogy is that it is predicated on the tool. Specifically, design possibilities are limited to a certain degree by the type of software used and the capabilities that the software has in relation to creating architectural form. Thanks to software packages such as Maya and Form Z, a new architectural language has emerged that challenges both the tenets of the discourse of architecture and standard construction means and methods. Not unexpectedly, projects built using this method tend to suffer from the abrupt translation from a digital to the physical realm.

While retaining a fascination with the fluid, we have applied the same digital parameters to understanding the dynamics of environmental forces that affect the built environment. Instead of modelling fluid forms that depict the circulation patterns of a site as Lynn does, we chose to simulate the fluid nature of water as it interacts with a building, so that the design may be improved accordingly. Ultimately we would hope to shape our chosen fluid, water, indirectly, by strategically shaping architectural form.

\section{Process}

One of the many paradoxes that digital tools present is the seemingly infinite operations that are achievable with such little effort. Though simple in premise, the task of digitally simulating rainfall in an accurate and measured way has proven to be remarkable challenging. Given the plethora of software that are available and their intrinsic complexities, it became immediately apparent that we would need to look far a partner with greater familiarity and programming expertise. For these reasons we forged a connection between our School and one of the University of Florida's premier interdisciplinary centers, the Digital Worlds Institute currently housed jointly in the colleges of Engineering and Fine Arts.

Realizing the institutional friction a formal collaboration might create, we began simply. Three architecture professors set aside a two-week segment within a design studio dedicated to building in the Florida landscape. Our 
climate (semi-tropical with daily rainfall all summer) makes a study of water obvious and, in retrospect, almost essential. We posed this question to the students and faculty in an ongoing special topics research seminar taught within the Digital Worlds Institute: Can the flow of rainfall onto a building and landscape be digitally simulated in such a way that students can understand the repercussions of their designs and then work to modify their structures to more responsibly and poetically shed water?

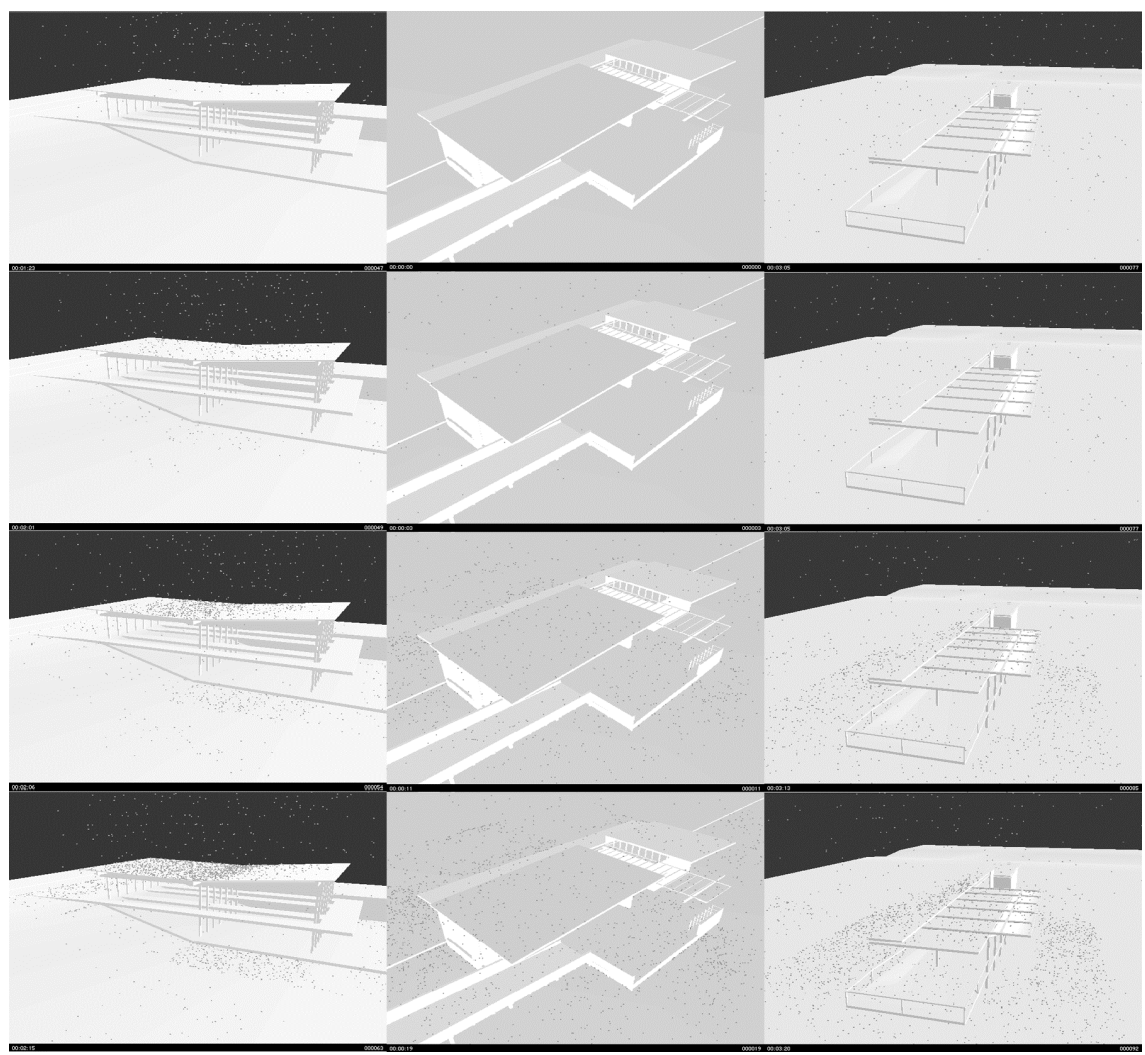

Figure 1: $\quad$ Captured stills from Maya.

After a series of discussions regarding software alternatives, Maya was selected as the testing platform, given its capacities to simulate gravitational behavior and impeding surfaces. With the assistance of the Digital Worlds Institute, the students were able to successfully import their virtual models into the dynamic animation environment found within Maya. Exported as .dxf files from 3D Studio Max and introduced to the Maya three-dimensional environment, the process for particle simulation was relatively simple. Maya allowed for the creation of a particle emitting source element placed above the model and a gravity element created below the model to control the direction of particle movement. This system established the preliminary condition of purely 
vertical particulate flow as an abstraction of individual raindrops falling. Density was modulated through a numerical system of randomly located particles emitted with an upward limit of 500 particles/second. Adjustments with particle size accounted for the approximate size of individual drops. Modulation of the particle's dynamic response to encountered surfaces allowed for an approximation of splash. Further definition within the model itself was required in order for the particles to react to the model surfaces directly. All modelled surfaces required definition as either an active (impenetrable) or inactive (penetrable) surface. An inactive surface would have no impact on the direction or rate of travel of the particles, particles simply passed through them. Active surfaces could alter both the direction and rate of descent of particles, determined by the orientation of the surface itself as well as adjustments within the friction characteristics assigned to that surface.

The product of this simulation was a short length digital animation displaying the idealized behavior of raindrops falling onto an architectural construct and landscape. Though numerically indeterminate, the animations did allow for a visual means of analysis regarding roof and site drainage within the specific limitations of this scenario. The animations clearly delineated the fundamental principles of fluid movement across varied planes that provided visual confirmation of general drainage patterns and collection points within the tectonic elements of the architecture as well as the diagrammatic conditions of site.

The process of development for this simulation prototype also revealed numerous difficulties specific to digital media, stemming primarily from software interaction. The most dominant struggle occurred with the interface between Maya and 3D Studio Max. The necessity of a plug-in for importing Max files was not initially known. Only after several days of struggle on the part of the DW was the software interface problem solved, and even then only with the addition of a secondary software element. Equally, the software interface appeared to only work with .dxf files written directly from Max. Though this was much less of a problem due to the relative ease of translation between the various modelling programs used by students, it still imposed an additional level of file translation as well as the possibility for file corruption or data loss.

Once the files were in Maya, a new set of challenges became apparent, ranging from software and hardware limitations to lack of familiarity by the user. Though the DW had been successful in projecting particles onto a surface and incurring an effective representation of gravitational flow, the behavior of the particles could not be adapted to fit the characteristics of fluid movement. The resulting simulations, though indicative of general flow patterns were more fictional at roof edges, where the particles followed a trajectory which was in fact noticeably longer in horizontal throw than would actually occur with liquid flow. Equally, the particles necessarily remained autonomous as elements, not interacting or combining with adjacent particles to form larger fluid bodies, which in turn allowed for only the suggestion of pooling water on a roof or a site. They never exhibited cohesion. While Maya 4.5 does have potential for the animation of fluid behavior, the lack of familiarity with the program effectively 
limited the simulation to a system of particle animation. Neither the students nor DW had enough working versatility within Maya to fully take advantage of the program's potential, let alone the possibility of innovative usage to achieve a specific type of effect.

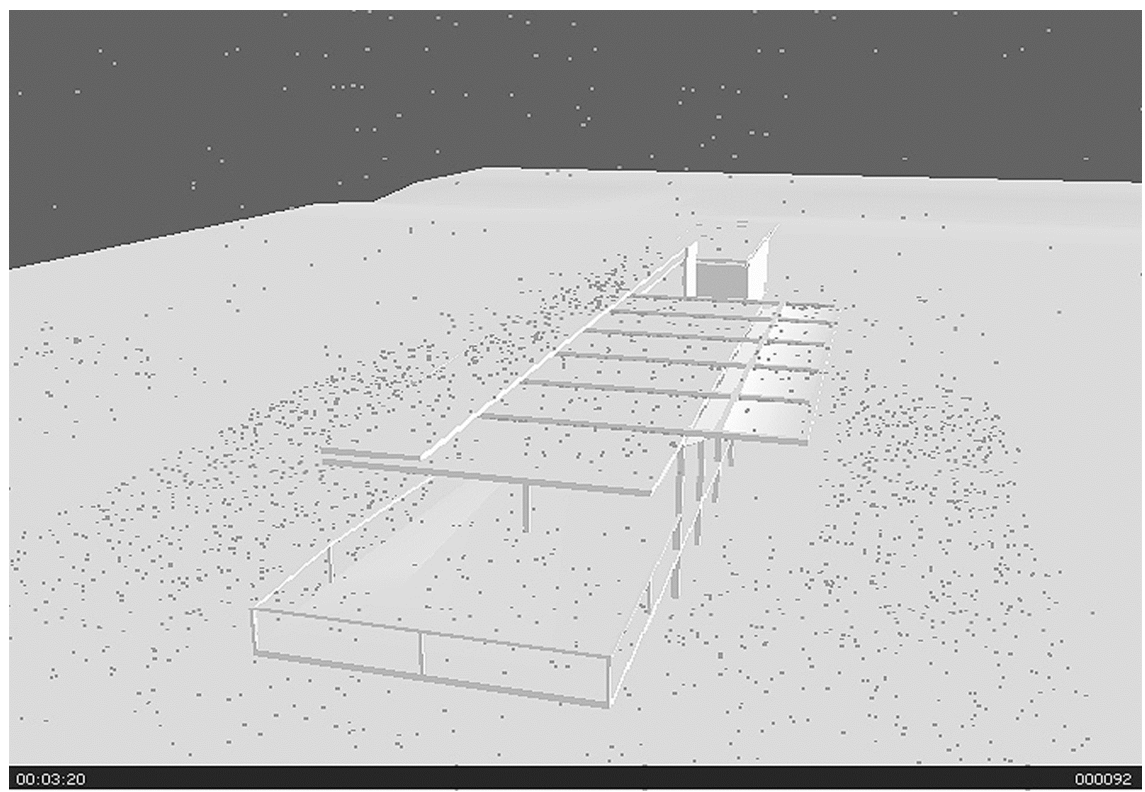

Figure 2: Designed project inserted into the particle simulation environment.

As Maya is primarily intended as animation effect software, the computational limitations became equally apparent. Though density of rainfall could be visually adjusted, rainfall intensity was effectively limited by the size and number of emitting elements. The choice to use multiple emitting elements would have increased the file size and complexity, and applied further strain to the processing capacity of the system. At the very least it would have substantially increased animation times, if it did not crash the entire system.

The particle emission rate was qualitatively inconvertible. Any specific calibration to a more common system of notation, such as inches per hour, could not be achieved. In addition, environmental factors such as constant or gusting winds could not be explored in the allotted time. The possibility of surface distinction was equally vague. Permeability of surfaces could be implied with adjusted friction, but the particles could not penetrate the surface, only move more slowly on it.

A further compounding struggle was the hardware itself. Maya's intensive operations require a powerful CPU in both processor speed and memory. Model complexity and thus accuracy had to be kept to a minimum in order to simplify the particle simulation. In addition, once the models were imported into Maya 4.5, all operations had to be performed with this version. Preceding 
versions of Maya were not compatible, resulting in a reduction of the available number of viable software seats.

Later experimentations involved Max exclusively instead of Maya due to the improved inter-changeability of file formats between programs. At the time, this hindrance proved quite time consuming and limited the ultimate outcome. Max directly imports the students' AutoCAD model files, and allows for a more logical assignment of material characteristics than Maya. Solid objects are assigned to be Rigid Bodies so that the particle system simulating rain cannot pass through them. Max also allowed for more control over the simulated rain. The amount, direction, intensity, size, and speed of rain drips are controllable through extensive parametric settings. These options are easily adjusted for different climatic situations, so one can test rain run off for simple rain showers as well as hurricanes and snowstorms if the need arises.

One of the new challenges with the research is to make the rain particles also act with fluid characteristics when they pool together. Though not essential to the project, the inability to transform the particles to fluids became somewhat of an elusive aspiration. Even with the lifespan for the particles set to a maximum value, once the particle ceased to move and collected together they resembled a somewhat gooey mass of ping-pong balls.

\section{Conclusions}

The collaboration recounted here highlighted the challenges of interdisciplinary exchange and the importance of intangible personal qualities and methods for problem solving. This experiment brought to light not only the strengths and limitations of the each of the disciplines involved, but also exposed the representational preferences of each. Given the architecture students' strengths with visualization, the modelling software interface proved highly effective, allowing for basic modulations and adjustments with intuitive operations within a three-dimensional environment. However, when the questions arose regarding file formatting, or specific value characteristics of a tool or operation, the architecture students quickly became frustrated. As a counterpoint to this method of thinking, the DW participants excelled with the intricacies and nuances of the software, departing from more intuitive methods of working in favour of a stronger methodology and numerical understanding of each operation.

We were gratified to see an emerging collaborative ethos among our students. Although the project initially privileged the knowledge base and technology housed within the Digital Worlds Institute, the architecture students' diligence, enthusiasm and expertise turned our team from the DW's "clients" into true collaborators. By the end of the two-week experiment our students were answering as many questions as they asked.

While this modest collaboration between distinct disciplines provided some preliminary cohesion - temporarily turning particulate disciplinary studies into a fluid exchange - our primary goal remains untested. Our objective is to use dynamic particle simulation to influence design decision-making. To date we 
have crudely modelled a fluid condition, but we have not yet helped our students use the properties of rain, our chosen fluid, to inform design.
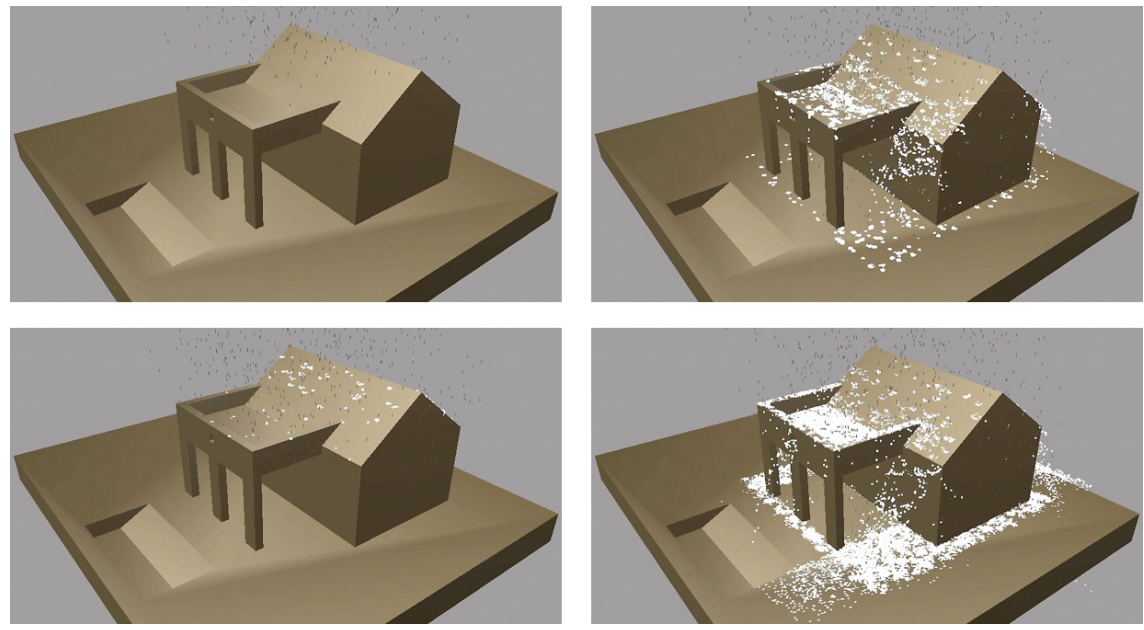

Figure 3: $\quad$ Further attempt of modelling rain with instanced blobby surfaces.

Finally, this project has been developed between individual faculty members outside of broader institutional support. In the long run, such collaborations can only be fully realized if they are offered stronger support from within each discipline, more fluidly integrating new proposals and investigations with existing intradisciplinary programs rather than the perpetuation of a more proprietary attitude. The potential of a truly collegial relationship between otherwise disparate disciples could help to offset the constrained fiscal environment most departments, schools, and universities operate within.

\section{References}

[1] Luis Barragan, quoted in Emilio Ambasz, The Architecture of Luis Barragan, The Museum of Modern Art, New York, 1976, p. 9

[2] William Gibson, Neuromancer, Ace Books, New York, 1984, pg. 51.

[3] Marcos Novak, Liquid Architectures in Cyberspace, First Steps MIT Press, 1991. pg. 251-2.

[4] Greg Lynn, Animate Form, Princeton Architectural Press, New York, 1999, pg. 103.

[5] Dodge, Martin and Kitchin, Rob Atlas of Cyberspace, Pearson Educational Press, London, 2001.

[6] Steven R. Holtzman, Digital Mantras: The Languages of Abstraction and Virtual Worlds, MIT Press, Cambridge, Massachusetts, 1994.

[7] Mitchell, M., City of Bits: Space, Place, and the Infobahn, MIT Press, Cambridge, Massachusetts, 1995.

[8] Rashid, Hani and Couture, Lise-Anne, Asymptote Flux, Phaidon Press Inc, NY, 2002. 